\title{
SCHMIDT'S ORTHOGONAL ENNUPLE AND THE FRENET FORMULAS FOR A CURVE
}

BY A. J. MC CONNELL

This note deals with $\$ 32$ (pp. 103-107) of Eisenhart's Riemannian Geometry.

The object of the above section is to define an orthogonal ennuple from a given set of $n$ linearly independent vectors and hence to deduce the Frenet formulas of a curve in a space of $n$ dimensions. One criticism of Eisenhart's ennuple is that when the original set of vectors forms an orthogonal ennuple the defined ennuple does not reduce to it, and in this note I have modified the definition so that this disadvantage is removed. Also, to obtain equations (32.18) in his book, Eisenhart has assumed that $b_{p-1}=\left(b_{p-1}^{2}\right)^{1 / 2}$, and this is incorrect if we understand that the positive root has been taken.

Being given a set of $n$ linearly independent vectors,

$$
\xi_{\alpha \mid}^{r} \quad(\alpha=1,2,3, \cdots, n),
$$

in a Riemannian manifold of $n$ dimensions, our object is to define from these vectors a set of $n$ orthogonal unit vectors,

$$
\lambda_{a-1}^{r}, \quad(\alpha=1,2,3, \cdots, n) .
$$

Building up the ennuple in the usual manner,* we arrive at the formulas

$$
\lambda_{p \mid}^{r}=\epsilon_{p}\left(\left|b_{p} / b_{p-1}\right|\right)^{1 / 2} \sum_{\alpha=1}^{p} \xi_{\alpha \mid}^{r} B_{p}^{\alpha}, \quad(p=1,2, \cdots, n) .
$$

The quantities $\epsilon_{p}$ determine the orientation of the ennuple in space. Eisenhart takes all the $\epsilon$ 's equal to +1 . I propose to choose them so that when (1) is an orthogonal ennuple the set (2) may reduce to it. We easily find that we must

* See Kowalewski, Determinantentheorie, pp. 423-426. 
put $\epsilon_{p}=e_{p}$, where $e_{p}$ has its usual meaning. Our orthogonal ennuple is therefore given by

$$
\lambda_{p \mid}^{r}=e_{p}\left(\left|b_{p} / b_{p-1}\right|\right)^{1 / 2} \sum_{\alpha=1}^{p} \xi_{\alpha \mid}^{r} B_{p}^{\alpha}, \quad(p=1,2, \cdots, n) .
$$

The Frenet formulas, as deduced from Eisenhart's form of the ennuple, are given by equations (32.16) and (32.18) of the above section. In the formulas (32.18) for the curvatures, Eisenhart errs in absorbing under a square root sign which calls always for the positive value a quantity which may be negative. The equations should read

$$
\frac{1}{\rho_{p}}=e_{p}\left(\left|b_{p-1} b_{p+1} / b_{p}{ }^{2}\right|\right)^{1 / 2} .
$$

In order to obtain the principal normals resulting from the ennuple (4), we need only substitute $e_{p} \lambda_{p \mid}^{r}$ for $\lambda_{p \mid}^{r}$ in (32.16) which will then read

(6) $\frac{d x^{m}}{d s}-\lambda_{p \mid, m}^{r}=\frac{e_{p}}{\rho_{p}} \lambda_{p+1 \mid} r-\frac{e_{p}}{\rho_{p-1}} \lambda_{p-1 \mid}, \quad(p=2, \cdots, n-1)$.

It may be preferable sometimes to have all the curvatures positive, as they obviously are in the above equations for a positive definite line-element. Thus, putting $1 / \rho_{p}=e_{p} k_{p}$, $k_{p}>0$, we have the Frenet formulas in the final form

$$
\begin{array}{r}
\frac{d x^{m}}{d s}-\lambda_{p \mid, m}^{r}=k_{p} \lambda_{p+11}-e_{p} e_{p-1} k_{p-1} \lambda_{p-1 \mid}, \\
(p=2, \cdots, n-1) .
\end{array}
$$

These are the normals and curvatures defined by Synge.* Of course, in the case of a positive definite line-element, the two sets of normals coincide.

Trintty College, Dublin

* Synge, Normals and curvatures of a curve in a Riemannian manifold, Proceedings of the International Mathematical Congress, Toronto, 1924. 